\title{
Longitudinal Performance Analysis in Ultra-Triathlon of the World's 2 Best Master Triathletes
}

\author{
Sousa, Caio Victor ; Knechtle, Beat ; Nikolaidis, Pantelis Theo
}

\begin{abstract}
Purpose: To analyze the performances of 2 ultra-triathletes who competed in ultra-triathlon events (double Iron ultra-triathlon and triple Iron ultra-triathlon) for the past 3 decades. Longitudinal data of the performance development in ultra-triathlon athletes spanning many years are rare. Prediction of age-related performance declines in the different disciplines in triathlon events (swimming, cycling, and running) are needed for race directors to set realistic goals (time limits) for master athletes in these events. Methods: Athletes A and B had 34 and 53 participations in double Iron at 35-55 and 40-69 y of age, respectively, and 26 and 20 participations in triple Iron at 33-51 and 40-61 y of age, respectively. Nonlinear regression analyses were performed with split and overall performance against age. Results: The average declines in performance in triple Iron ultra-triathlon for athlete A were $0.62 \% / y, 0.19 \% / y$, and $0.98 \% / y$ for swimming, cycling, and running, respectively. For athlete B, a positive change was identified for swimming $(0.19 \% / y)$ and cycling $(1.12 \% / y)$ but negative change for running $(1.34 \% / y)$. Conclusion: Running is the discipline with the greatest performance-decline rate for both athletes, in both double and triple Iron distances. The race time limit of double Iron competitions seems too short, making it difficult for master athletes older than $55 \mathrm{y}$ to finish the race within the event regulations.
\end{abstract}

DOI: https://doi.org/10.1123/ijspp.2019-0805

Posted at the Zurich Open Repository and Archive, University of Zurich

ZORA URL: https://doi.org/10.5167/uzh-189909

Journal Article

Accepted Version

Originally published at:

Sousa, Caio Victor; Knechtle, Beat; Nikolaidis, Pantelis Theo (2020). Longitudinal Performance Analysis in UltraTriathlon of the World's 2 Best Master Triathletes. International Journal of Sports Physiology and Performance, 15(10):1480-1484.

DOI: https://doi.org/10.1123/ijspp.2019-0805 


\section{Longitudinal performance analysis in ultra-triathlon of the world's two best master triathletes}

Brief report / Case study

Caio Victor Sousa ${ }^{1}$

cvsousa89@gmail.com

Beat Knechtle ${ }^{2,3}$

beat.knechtle@hispeed.ch

Pantelis Theo Nikolaidis ${ }^{4}$ pademil@hotmail.com

${ }^{1}$ College of Arts, Media \& Design; Bouve College of Health Sciences, Northeastern University, Boston, USA

${ }^{2}$ Medbase St. Gallen Am Vadianplatz, St. Gallen, Switzerland

${ }^{3}$ Institute of Primary Care, University of Zurich, Zurich, Switzerland

${ }^{4}$ Exercise Physiology Laboratory, Nikaia, Greece

\section{Corresponding author}

Prof. Dr. med. Beat Knechtle

Medbase St. Gallen Am Vadianplatz

Vadianstrasse 26

9001 St. Gallen

Switzerland

Telephone $\quad+41(0) 712269300$

Telefax +41(0) 712269301

E-Mail: beat.knechtle@hispeed.ch

Preferred running head: Performance analysis of ultra-triathlon

Abstract word count: 215

Text-only word count: 1988

Number of figures: 3 


\section{Abstract}

Purpose: This case study analyzed the performances of two ultra-triathletes who competed in ultra-triathlon events (Double and Triple Iron ultra-triathlon) for the past three decades.

35 Longitudinal data of the performance development in ultra-triathlon athletes spanning many 36 years are rare. Prediction of age-related performance declines in the different disciplines in 37 triathlon events (swimming, cycling, and running) are needed for race directors to set realistic 38 goals (time limits) for master athletes in these events.

39 Methods: Athletes A and B had 34 and 53 participations in Double Iron with 35-55 and 40-

4069 years of age, respectively; and 26 and 20 participations in Triple Iron with 33-51 and 40-

4161 years of age, respectively. Non-linear regression analyses were performed with split and

42 overall performance against age.

43 Results: The average declines in performance in Triple Iron ultra-triathlon for Athlete A were $440.62 \% / y e a r, 0.19 \% / y e a r$ and $0.98 \% / y e a r$ for swimming, cycling and running, respectively.

45 For Athlete B, it was identified a positive change for swimming $(0.19 \% / y e a r)$ and cycling 46 (1.12\%/year) and negative for running (1.34\%/year).

47 Conclusion: Running is the discipline with the greatest performance declines rate for both 48 athletes, in both double and triple-Iron distances. The race time limit of Double Iron 49 competitions seems too short, making it difficult for master athletes older than 55 years old to 50 finish the race within the event regulations.

52 Keywords: endurance, performance, swimming, cycling, running 


\section{INTRODUCTION}

It is well known that athletic performance declines with increasing age ${ }^{1}$. This has been shown for different sports disciplines such as swimming ${ }^{2}$, running ${ }^{1}$ and multi-sports disciplines such as triathlon with the combination of swimming, cycling and running ${ }^{3-5}$.

The age-related performance decline in triathlon has been investigated for Olympic distance triathlon ${ }^{3,5}$, for Ironman triathlon (i.e. $3.8 \mathrm{~km}$ swimming, $180 \mathrm{~km}$ cycling, and $42.195 \mathrm{~km}$ running $)^{5}$, and for longer distances such as the Triple Iron ultra-triathlon and the Deca Iron ultra-triathlon ${ }^{4}$. It has been shown that the age-related decline in triathlon performance is specific to the discipline ${ }^{5,6}$. For Olympic and Ironman distance triathlon, cycling showed fewer declines in performance with age than swimming and running 5 .

Studies investigating the age-related performance decline in triathlon used a cross-sectional design investigating large samples of athletes. Sousa, Nikolaidis, Knechtle ${ }^{7}$ investigated the performance trends in ultra-triathlons from 1985 to 2018 and reported differences the time spent in the three disciplines depending on the sex and distance of the race, and also that men showed a decrease in performance throughout the years. However, although this study had a time effect on performance, it was a cross-sectional analysis with multiple athletes through several years. The longitudinal analysis of the age-related performance decline in endurance sports is, however, rather rare $^{8}$ and missing for triathlon. The outcome of such results throughout the years might be useful for other athletes and coaches to predict future performance changes due to increasing age and also to race directors to set realistic goals (i.e. time limits) for master athletes.

Therefore, the first aim of the present case study was to determine the age-related performance decline in a longitudinal analysis of race performances including performance in split disciplines (i.e. swimming, cycling and running). The second aim was to extrapolate the race performance in order to define when the athletes would be no longer able to finish a race (i.e. Double Iron ultra-triathlon covering $7.6 \mathrm{~km}$ swimming, $360 \mathrm{~km}$ cycling and $84.4 \mathrm{~km}$ running and Triple Iron ultra-triathlon covering $11.4 \mathrm{~km}$ swimming, $540 \mathrm{~km}$ cycling and $126.6 \mathrm{~km}$ running) within the official time limit.

\section{METHODS}

\section{Ethical approval}

This study was approved by the Institutional Review Board of Kanton St. Gallen, Switzerland, with a waiver of the requirement for informed consent of the participants as the study involved the analysis of publicly available data. The study was conducted in accordance with recognized ethical standards according to the Declaration of Helsinki adopted in 1964 and revised in 2013.

\section{Study design}

The study is a retrospective cohort study with longitudinal data from over 20 years of two master athletes. We analyzed the age-related performance decline in swimming, cycling, running and overall race time in two of the world's best ultra-triathletes having completed each more than 200 ultra-triathlons (i.e. longer than the Ironman distance) over more than 20 years.

\section{Race events}


The race events are all organized by the International Ultra Triathlon Association (IUTA), a non-profitable organization founded in 1992 which current member are a majority of previous or current ultra-triathletes, making it easy to organizers to know what it takes to get to the

\section{6 finish line.}

107

Each race starts at 7 a.m. with 10 to 40 athletes (personal selection by the race director based upon earlier achievements). Swimming is generally held in pools $(50 \mathrm{~m})$ or lakes with laps of 400 to $500 \mathrm{~m}$. Cycling and running are generally held in a closed circuit with up to $10 \mathrm{~km}$ and $1-2 \mathrm{~km}$, respectively. Each athlete has his own support crew for nutrition, change of clothes and equipment.

In each kind of event (Double or Triple Iron), the athletes have to swim first the full distance of 7.6 or $11.4 \mathrm{~km}$, respectively, to cycle the full distance of 360 or $540 \mathrm{~km}$, respectively, and to run the full distance of 84.4 or 12.6. $\mathrm{km}$. The time limit for a Double Iron ultra-triathlon depends upon the race director and is at 34 hours (e.g., Double Iron Germany, Double Iron Lithuania) or 36 hours (e.g., Double Iron Florida, Double Iron Virginia). For a Triple Iron ultra-triathlon, the time limit is at 58 hours (e.g., Triple Iron Germany) or 60 hours (e.g., Triple Iron Austria, Triple Iron Virginia).

Together with all the physiological and strategic challenges of such events, ultra-triathletes also have good psychological skills to finish the races. The majority of the race is performed in low intensities in a repetitive course. Thus, a very unique mindset is developed to avoid boredom and to maintain focus.

\section{Athletes}

Among all athletes who have ever finished an ultra-triathlon event organized by IUTA, two athletes have finished more than 200 ultra-triathlons and numerous other ultra-triathlon events listed in this article. For the purpose of this study, all race results (i.e. overall race times and split times in swimming, cycling and running) were collected by both athletes, this since the first edition of an ultra-triathlon (i.e. a triathlon longer than the classical Ironman distance). All data are publicly available on IUTA's website ${ }^{9}$. For the two athletes, all split and overall race times for both a Double and Triple Iron ultra-triathlon were recorded.

Athlete B has finished most Double Iron ultra-triathlons ever while athlete A has finished most Triple Iron ultra-triathlons ever. Available data for Double Iron from Athlete A and B were from 35-55 and 40-69 years old, respectively; and 33-51 and 40-61 years old for Triple Iron.

The two triathletes were electronically interviewed and asked to provide information about their experience and training routine along the years. Both have more than 20 years of experience in endurance training, particularly triathlon. The season for these events is usually from March to November (race event), with the large portion of trainings happening between April and October. Athlete A recorded his training since 1995. In a regular year, Athlete A expended an average of 1.4 weekly hours of swimming, 16.4 weekly hours of cycling, and 7.3 weekly hours of running. When during preparation for the competition (April-October) this numbers raises to 1.7 weekly hours of swimming, 22.1 weekly hours of cycling, and 10.1 weekly hours of running, which represents $5.1 \%, 65.2 \%$ and $29.7 \%$ of total training hours, respectively. This represent long volumes and low intensity sessions.

\section{Statistical Analysis}


153 Non-linear regressions ( $2^{\text {nd }}$ order, polynomial) were applied in split performances (swimming, 154 cycling and running) versus age for both athletes in Double and Triple Iron. Additionally, 155 non-linear regressions ( $2^{\text {nd }}$ order, polynomial) were also applied for their overall race times in 156 Double and Triple Iron ultra-triathlon versus age, and the generated equation were used to 157 estimate an age which the overall performance (race time) would be above the race time limit. 158 All procedures were conducted using Microsoft Office Excel 2019 and GraphPad Prism v8.2. 


\section{RESULTS}

For Double Iron, Athlete A had an average performance loss over time of $0.34 \% / y e a r$, $0.19 \% / y e a r$ and $0.82 \% / y e a r$ for swimming, cycling and running, respectively. Athlete B had an average performance loss of $0.42 \%$ /year, $0.49 \% / y e a r$ and $0.69 \% / y e a r$ for swimming, cycling and running, respectively (Figure 1).

\section{Figure 1}

The average declines in performance in Triple Iron ultra-triathlon for Athlete A were $0.62 \% / y e a r, 0.19 \% / y e a r$ and $0.98 \%$ /year for swimming, cycling and running, respectively. For Athlete B, it was identified a positive change for swimming (0.19\%/year) and cycling (1.12\%/year) and negative for running (1.34\%/year; Figure 2$)$.

\section{Figure 2}

Non-linear regressions for the overall results showed that both athletes have been reducing their performance in Double Iron ultra-triathlon with a reduction by $0.68 \% / y e a r$ and $0.84 \% / y e a r$ for athletes A and B, respectively. Furthermore, the non-linear regression to predict the possible age when the athlete would not be able to finish a Double Iron ultratriathlon before the time-limit was 55 and 60 years old (Figure 3). For Triple Iron ultratriathlon, the nature of the data does not allow to perform a model to predict the possible 'agelimit'. Athlete A had increased his overall race time in the first decade, it remained relatively stable afterwards. Whereas Athlete B had a slight increase in overall race time across each year.

\section{Figure 3}

\section{DISCUSSION}

The main findings of the present manuscript were that the running split is the discipline where both athletes showed the highest performance decline rate in both Double and Triple Iron distances. Additionally, it is surprising to note a performance increase in one of the athletes for the swimming and cycling split in the Triple Iron ultra-triathlon. Ultimately, overall performance showed that after the age of 55 years old it becomes very hard to finish the event within the race time limit for the Double Iron distance, but not for the Triple Iron.

The running discipline is the triathlon split with the greatest age-related decline in almost all triathlon distances ${ }^{3,4,6}$. Due to biomechanical characteristics, running has a greater risk of injury when compared to cycling or swimming ${ }^{10}$, which may limit the amount of training undertaken in running compared to swimming and cycling ${ }^{2}$. In addition, a lesser reduction in lactate threshold or economy during cycling, or a greater muscular fatigue during running with aging have been also proposed as potential explanations ${ }^{11}$.

The performance declines in swimming and cycling can be attributed to an age-related reduction of skeletal muscle mass and a decline in muscle and cardiorespiratory effectiveness ${ }^{12}$. Although experience seems to be a key factor to success in ultra-endurance events $^{4}$, the natural age-related physiological decline seems to be irreversible in Double Iron ultra-triathlon. 
Regarding the Triple Iron ultra-triathlon distance, both athletes reduced their overall performance throughout the years. On the one hand, athlete A had an increase in overall race time in the first decade and remained relatively stable afterwards; on the other hand, athlete B had a slight increase in race time performance in the two decades. Evidence shows that the longer the distance in ultra-triathlon, the longer the influence of experience in overall performance ${ }^{4}$. Additionally, a change of strategy can be observed in athlete $\mathrm{B}$, that in average performed better in both swimming and cycling despite the increasing age. Observing a transient improvement in an elite master triathlete would not be surprising considering data on triathlon performance across calendar years ${ }^{13}$. According to these data, an improvement in performance was shown in master triathletes over the 1995-2010 period, which might be due to technical and scientific advances.

220

221

222

223

224

225

226

227

228

229

230

231

232

233

234

A physiological approach to the overall age-related performance decline can be attributed to several factors: reduction in the activity of the hypothalamic-pituitary-gonadal axis ${ }^{14,15}$, making it difficult to maintain muscle mass and function; decrease in pulmonary function ${ }^{16}$; accumulation of DNA damage ${ }^{17}$, due to increased oxidative stress and chronic inflammation ${ }^{18}$; changes in running kinematics ${ }^{19}$, possibly reducing their efficiency and running economy. These factors combined reflects in their recovery after each training session, possibly leading to a reduction in training volume and number of more intensive training sessions ${ }^{20}$. Although evidence shows that endurance athletes are less biologically aged as their non-athlete peers ${ }^{21}$, their natural aging process reverberates in their physical performance throughout the years as was clearly reflected in the present results.

\section{PRACTICAL APPLICATIONS}

Master athletes usually have a very healthy lifestyle, which is a key aspect for them to be able to keep competing in sports events. Nevertheless, they are not immune to the age-related physiological decline, which affects their performance and it seems that may preclude them to participate in some ultra-endurance events. Therefore, the present analysis may also be useful for race directors to set realistic goals in race time limit for master athletes. Specific race time limits based on the age group should be considered. Additionally, coaches and athletes could also use this analysis for long-term planning, considering weak spots and predicting which discipline could preclude an athlete to finish/participate in an ultra-triathlon.

\section{CONCLUSION}

In conclusion, running is the discipline with the greater performance decline for both athletes in both Double and Triple Iron ultra-triathlon distances. Additionally, it seems that the current rulebook of the Double Iron ultra-triathlon may have a too short race time limit, which would make it difficult to master athletes older than 55 years old to finish the race within the event regulations. 


\section{REFERENCES}

1. Ganse B, Ganse U, Dahl J, Degens H. Linear Decrease in Athletic Performance During the Human Life Span. Frontiers in physiology. 2018;9:1100.

2. Lepers R, Stapley PJ, Cattagni T. Variation of Age-Related Changes in Endurance Performance Between Modes of Locomotion in Men: An Analysis of Master World Records. Int J Sports Physiol Perform. 2018;13(3):394-397.

3. Etter F, Knechtle B, Rust CA, Rosemann T, Lepers R. The age-related decline in Olympic distance triathlon performance differs between males and females. The Journal of sports medicine and physical fitness. 2013;53(3):261-267.

4. Knechtle B, Rust CA, Knechtle P, Rosemann T, Lepers R. Age-related changes in ultra-triathlon performances. Extrem Physiol Med. 2012;1(1):5.

5. Lepers R, Sultana F, Bernard T, Hausswirth C, Brisswalter J. Age-related changes in triathlon performances. International journal of sports medicine. 2010;31(4):251-256.

6. Kach IW, Rust CA, Nikolaidis PT, Rosemann T, Knechtle B. The Age-Related Performance Decline in Ironman Triathlon Starts Earlier in Swimming Than in Cycling and Running. J Strength Cond Res. 2018;32(2):379-395.

7. Sousa CV, Nikolaidis PT, Knechtle B. Ultra-triathlon-Pacing, performance trends, the role of nationality, and sex differences in finishers and non-finishers. Scand J Med Sci Sports. 2019.

8. Stones MJ, Kozma A. Longitudinal trends in track and field performances. Exp Aging Res. 1984;10(2):107-110.

9. IUTA. Hall of Fame. International Ultra-Triathlon Association. www.iutasport.com/records/hall-of-fame-100. Published 2019. Accessed 10/09/2019, 2019.

10. Vleck V, Millet GP, Alves FB. The impact of triathlon training and racing on athletes' general health. Sports Med. 2014;44(12):1659-1692.

11. Lepers R, Knechtle B, Stapley PJ. Trends in Triathlon Performance: Effects of Sex and Age. Sports Med. 2013;43(9):851-863.

12. Tanaka H, Seals DR. Age and gender interactions in physiological functional capacity: insight from swimming performance. J Appl Physiol (1985). 1997;82(3):846-851.

13. Stiefel M, Knechtle B, Lepers R. Master triathletes have not reached limits in their Ironman triathlon performance. Scand J Med Sci Sports. 2014;24(1):89-97.

14. Kaufman JM, Vermeulen A. Declining gonadal function in elderly men. Bailliere's clinical endocrinology and metabolism. 1997;11(2):289-309.

15. Harman SM, Metter EJ, Tobin JD, Pearson J, Blackman MR, Baltimore Longitudinal Study of A. Longitudinal effects of aging on serum total and free testosterone levels in healthy men. Baltimore Longitudinal Study of Aging. J Clin Endocrinol Metab. 2001;86(2):724-731.

16. Degens H, Maden-Wilkinson TM, Ireland A, et al. Relationship between ventilatory function and age in master athletes and a sedentary reference population. Age (Dordr). 2013;35(3):1007-1015.

17. Cortopassi GA, Wang E. There is substantial agreement among interspecies estimates of DNA repair activity. Mechanisms of ageing and development. 1996;91(3):211-218.

18. Aguiar SS, Sousa CV, Deus LA, et al. Oxidative stress, inflammatory cytokines and body composition of master athletes: The interplay. Exp Gerontol. 2020;130:110806.

19. Dahl J, Degens H, Hildebrand F, Ganse B. Age-Related Changes of Sprint Kinematics. Front Physiol. 2019;10:613.

20. Kusy K, Zielinski J. Sprinters versus long-distance runners: how to grow old healthy. Exerc Sport Sci Rev. 2015;43(1):57-64. 
21. Sousa CV, Aguiar SS, Santos PA, et al. Telomere length and redox balance in master

301 endurance runners: The role of nitric oxide. Exp Gerontol. 2018. 
303

304

305

306

307

308

309

310

311

312

313

314

315

316

317

318

\section{Figure captions}

Figure 1. Split performance of swimming, cycling and running in double-Iron ultra-triathlon across the years from the two best ultra-triathletes still active. Y axis indicates the average speed in each discipline.

Figure 2. Split performance of swimming, cycling and running in triple-Iron ultra-triathlon across the years from the two best ultra-triathletes still active. Y axis indicates the average speed in each discipline.

Figure 3. Overall race time performance in double- and triple-Iron ultra-triathlon across the years from the two best ultra-triathletes still active. Dotted line represents the race time limit which may vary depending on the race location.

Figure 1
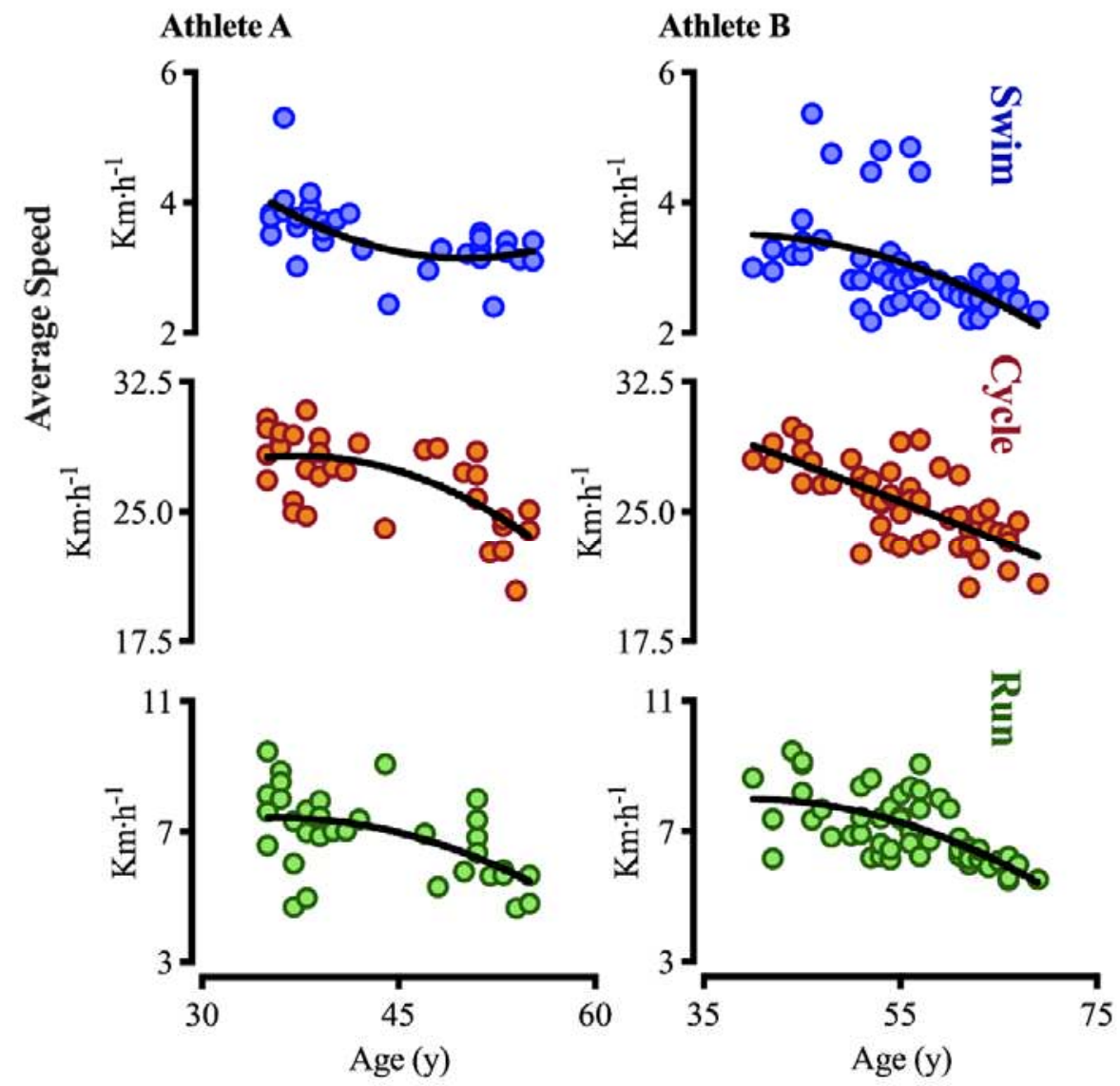

320

321

Figure 2 


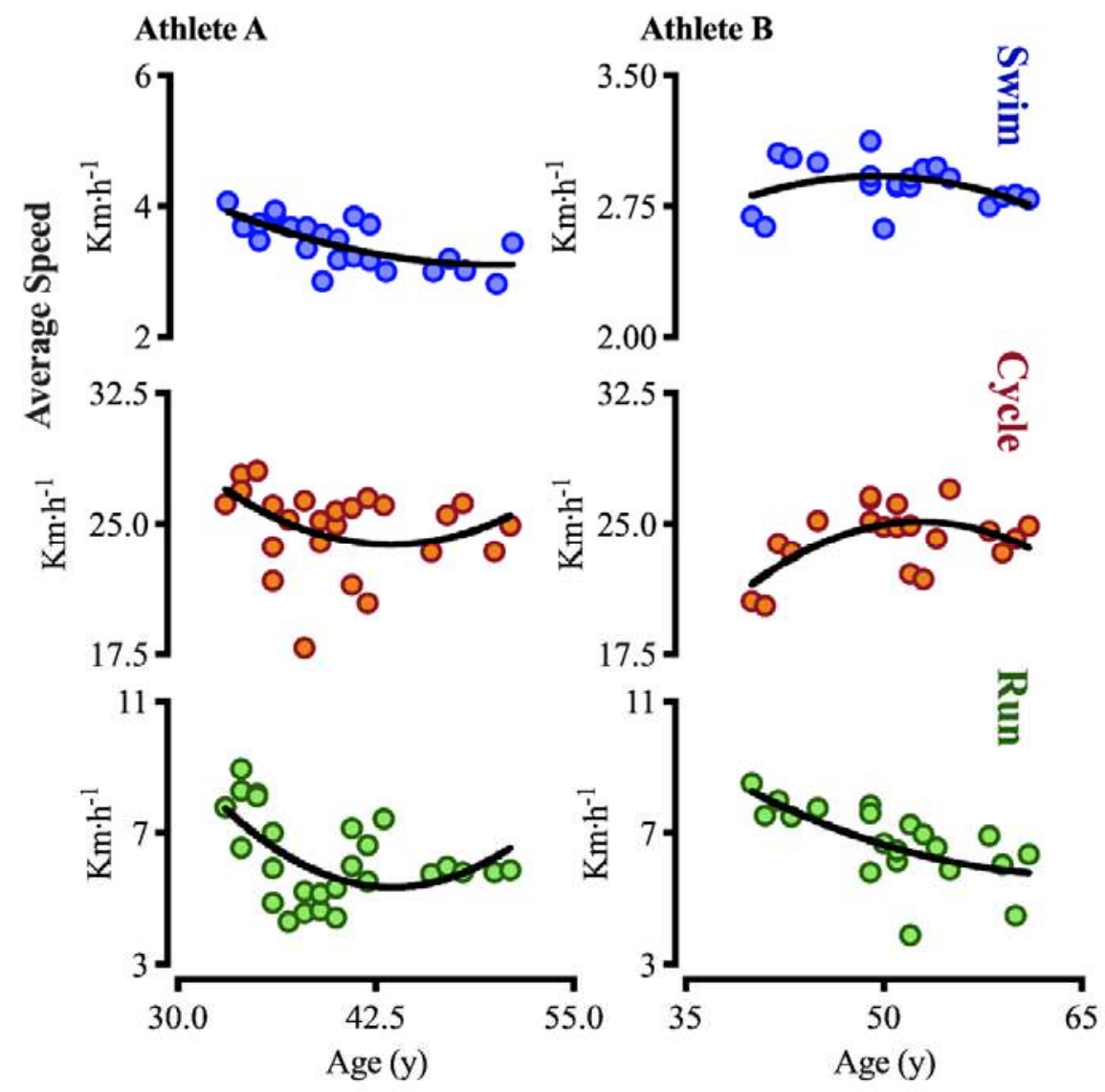

Figure 3 
Double-Iron
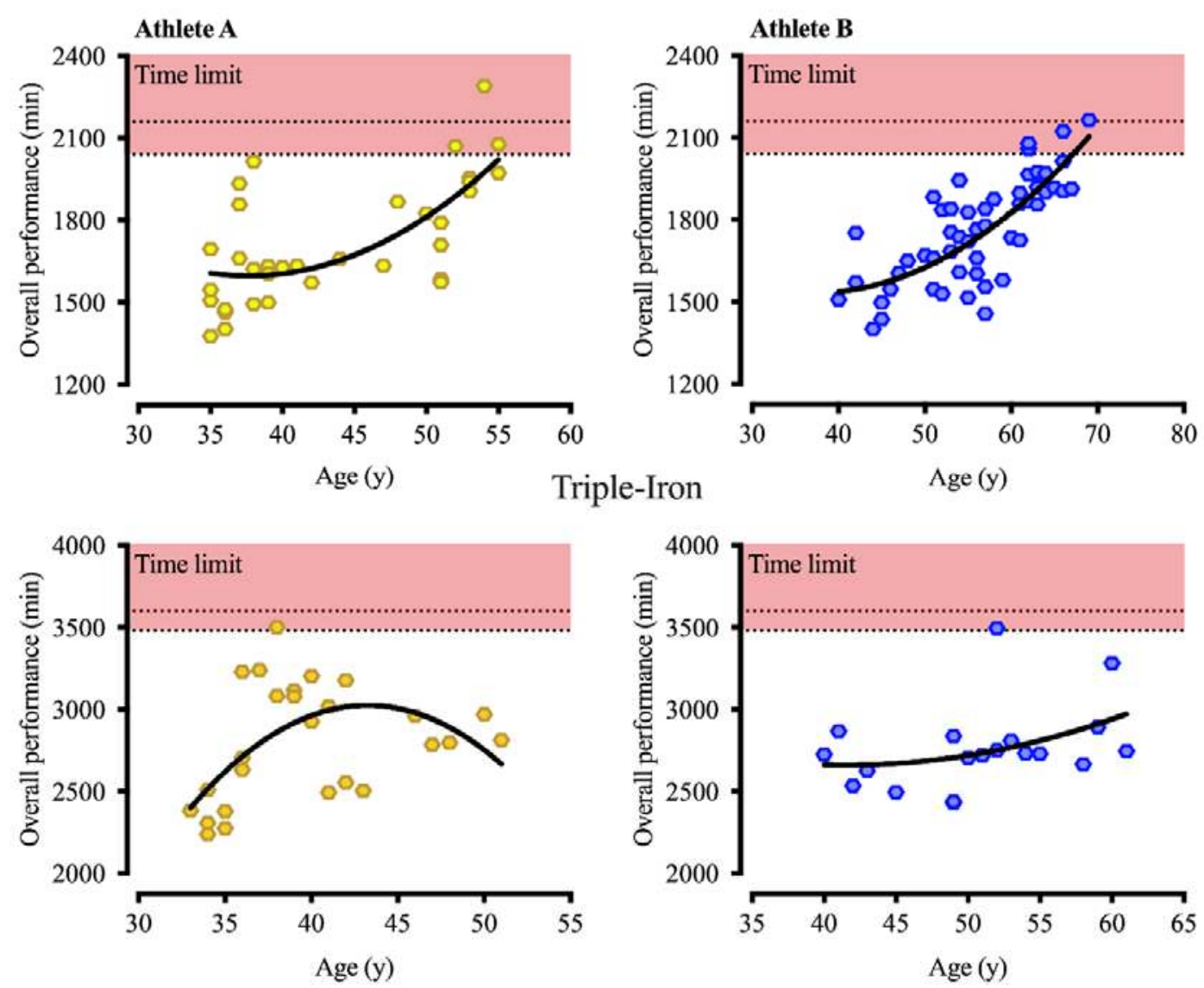